\title{
Regionale Pfarrblätter in der deutschen Schweiz
}

\author{
von Walter Ludin
}

Da in der Schweiz die Bistumszeitungen fehlen, haben hier die Pfarrblätter eine umfassendere publizistische Aufgabe als in der Bundesrepublik. Doch erst in den letzten paar Jahren wurden an verschiedenen Orten die personellen und finanziellen Mittel bereitgestellt, damit sie ihre Funktion auch wirklich erfüllen können. So gibt es - neben nach wie vor dürftigen „frommen Blättchen" - seit Beginn der 70er Jahre einige regional aufgebaute Pfarrblätter, die respektable publizistische Leistungen erbringen.

Betraditet man den "Pfarrblätterwald“ der (deutschen) Schweiz etwas näher, lassen sich etwa folgende Typen unterscheiden:

- „Vermeldblatt" mit kurzem, ein- bis anderthalbseitigem Vorspann (in der Regel ein einziger, konzeptionslos ausgewählter Artikel);

- „Vermeldblatt“ mit einer kleinen Sammlung kurzer Artikel (Informationen aus Region und Bistum, Lebenshilfen, Meditationen usw.);

- „Pfarrzeitschrift“ mit Reportagen, feuilletonistischen Beiträgen usw., wobei sich die Thematik praktisch ausschließlich auf die betreffende Pfarre konzentriert;

- „Rudimentärzeitschrift“ mit zwei bis drei Seiten erbaulichen Artikeln und ein bis zwei Seiten Pfarrmeldungen;

- „Magazine" mit einer Vielfalt von Themen und Gestaltungsformen.

Die neu gegründeten regionalen bzw. kantonalen Pfarrblätter (z. B. von Basel, Zürich, Bern) entsprechen dem letzten Typus. Eine Untersuchung der Aufgaben, die die kircheneigene Presse zu erfüllen hat, zeigt, daß die Lösung eindeutig bei dieser Form von Pfarrblättern liegt.

\section{Aufgaben der Pfarrblätter}

\section{Lokale Integration}

In erster Linie sollen die Pfarrblätter eine "gemeindebildende $\mathrm{Kraft}^{\text {"1 }}$ sein. Ahnlich wie die Lokalzeitungen schaffen sie auf der Pfarrebene lokale Integration. Als Bindeglied unter den Gläubigen tragen sie zum Pfarrbewußtsein bei ${ }^{2}$. Da die modernen Pfarren wegen ihrer Größe in vielen Fällen unüberschaubar geworden sind, müssen die herkömmlichen Kommunikationsformen (face to face) durch Kommunikationsmedien ergänzt werden, die mithelfen, „örtliche Offentlichkeit“ herzustellen.

Walter Ludin ofmcap studierte Journalistik in Freiburg (Schweiz). Er ist freier Mitarbeiter verschiedener Zeitungen und Verfasser einer Studie über "Aufgabe der Pfarrblätter in der kirchlichen Kommunikation". 
Es liegt auf der Hand, daß es zur Erfüllung dieser lokalen Integrationsaufgabe nicht genügt, die Pfarrblätter bloß mit Zahlen und Stichworten rein organisatorischer Aspekte (Gottesdienstzeiten, Jahrgedächtnisse usw.) zu füllen. Ihre Gestaltung darf nicht als bürokratische Aufgabe dem Pfarrsekretariat übertragen werden.

Die Pfarrblätter können die Aktivitäten und Probleme der Pfarren widerspiegeln, etwa durch Beiträge zu folgender Thematik:

- Auf Pfarrangebote in den verschiedensten Bereichen hinweisen wie Liturgie, Jugendarbeit und Sozialarbeit; damit solche Hinweise werbend wirken, dürfen sie nicht nur die nüchternen Angaben enthalten, die jene brauchen, die ohnehin schon bereit sind, darauf einzugehen.

- Verständnis wecken für Neuerungen in Liturgie, Religionsunterricht u. ä.; die Erfahrungen mit der Liturgiereform haben eindrucksvoll gezeigt, daß Neuerungen, die ohne Erklärung die Gläubigen überrumpeln, schlecht aufgenommen werden.

- Längerfriste Pläne vorstellen: Wenn die Gläubigen für das Leben der Pfarre mitverantwortlich sind, dürfen wichtige Entscheide nicht allein den verantwortlichen Gremien (Pfarreirat, Kirchgemeinderat) überlassen werden, ohne daß alle vorher mitdenken und mitberaten können.

- Uber Sitzungen offizieller Gremien sowie von Vereinen und Gruppen berichten: In lebendiger Form (keine trockenen Protokolle oder Communiqués!) sollen jene Informationen weitergegeben werden, die eine größere pfarrliche Offentlichkeit interessieren.

- Ein persönliches Wort des Pfarrers bringen: Der Pfarrer soll die Gelegenheit wahrnehmen, seine Anliegen regelmäßig in einer Art Kolumne darzulegen; solche persönlich gehaltenen Beiträge werden auch von jenen geschätzt, die kaum mit ihm in persönlichem Kontakt stehen.

- Pfarrchronik („Familiennachrichten“) wiedergeben: Mitteilungen über Taufen, Eheschließungen, Geburtstage, Todesfälle u. ä.

\section{Regionale Integration}

Das Leben der heutigen Menschen spielt sich nicht mehr im isolierten, eng begrenzten Raum der Gemeinde ab (Pendler, Shopping-Centers usw.!). Der regionale Lebensraum nimmt an Bedeutung zu. Da der Lebensraum auch zum Seelsorgeraum werden soll, muß das Pfarrprinzip durch das Regionalprinzip ergänzt werden.

Auch theologisch gesehen genügt die einzelne Pfarre sich selbst nicht. Sie ist auf das Größere der Diözese und durch sie auf die Gesamtkirche verwiesen. In der Praxis findet sich ansatzweise schon längst ein gestufter Seelsorgeaufbau, in dem die einzelnen Pfarren zu Dekanaten zusammengefaßt sind. Auf noch höherer Ebene bilden die Bischöfe eines Landes die nationalen Bischofskonferenzen.

Sollen die Pfarrblätter in der Lokalkirche eine Integrationsfunktion wahrnehmen, müssen sie auch den regionalen Gegebenheiten Rechnung tragen. Für Veranstaltungen, die auf die Gläubigen einer ganzen Region ausgerichtet sind, sind sie - zumindest etwa in der Schweiz - unersetzliche Publikationsmittel. So kann hier für Vorträge, Kurse oder Gottesdienste spezieller Zielgruppen geworben werden. Sozial- und andere Dienststellen, die einer ganzen Region zur Verfügung stehen, können auf ihre 
Angebote aufmerksam machen. Dadurch werden Bestrebungen regionaler Kooperation wirksam gefördert.

Analoge Aufgaben wie auf der Ebene der Pfarre und der Region wären für die Bistumsebene zu erwähnen. Schließlich sei noch daran erinnert, daß in den Pfarrblättern auch der Bezug zur Weltkirche nicht zu kurz kommen darf, selbst wenn der Schwerpunkt der Information und Kommunikation eindeutig auf der lokalen und regionalen Ebene liegt. Dabei besteht die Chance zur "Lokalisierung“ von Fragen, die außerhalb des eigenen Raums aktuell geworden sind ${ }^{3}$.

\section{Diskussions-Forum}

Spätestens seit dem Zweiten Vatikanischen Konzil hat der Pluralismus in der katholischen Kirche Fuß gefaßt. Sollen die verschiedenen Auffassungen und Glaubenshaltungen nicht zur Kirchenspaltung führen, ist das Gespräch zwischen den innerkirchlichen Gruppen nötig. Nur wenn jede Seite sich frei ausdrücken kann, führt der Pluralismus nicht zur Polarisierung. Der Dialog in Form des "ungehinderten Prozesses öffentlicher Meinungsbildung" kann einen Weg zur „Einheit und Gemeinsamkeit des Handelns" öffnen ${ }^{4}$. Ein "permanentes Glaubensgespräch“" soll entstehen.

Hier liegt eine große Chance der Pfarrblätter. Sie wird jedoch nur dann wahrgenommen, wenn die Redaktionen sich nicht aus Furcht vor Reaktionen auf eine Strategie der mittleren Linie zurückziehen und extreme Standpunkte von vornherein harmonisieren. Was außerhalb dieser Toleranzbreite läge, würde dann in das Getto separater Kommunikationsmittel verbannt. Statt daß die Polarisierung abgebaut würde, nähme sie zu.

Der Redakteur sollte sich nicht als Zensor "extremer" Meinungen betrachten, sondern als Animator des "Zeitgesprächs der Gesellschaft ${ }^{c}$. Als Animator der freien Diskussion soll er die Stummen zum Sprechen bringen. Er sollte den Mut haben, heiße Eisen anzupacken. Gerade anhand aktueller „Fälle" kann das öffentliche Interesse genutzt werden, um am konkreten Beispiel zu einem besseren Verständnis grundsätzlicher Sachverhalte beizutragen, sofern das theologische Hintergrundmaterial hinzugeliefert wird.

In der Praxis wird die offene Auseinandersetzung vor allem dadurch erschwert, daß vornehmlich die traditionellen Kreise oft wenig an einer offenen Diskussion interessiert sind. Sie ziehen die Polemik in ihren eigenen Medien meist vor.

Auch eine Reihe schweizerischer Pfarrblattredakteure hat noch etwas Mühe, von einem harmonisierenden Kurs der Mitte abzukommen. Daneben gibt es jedoch einige Redaktionen, die bewußt einen unzensurierten Dialog anstreben. Bereits die Planungsgruppe des neuen Pfarrblatts für den Kanton Bern stellte in ihrem Konzeptentwurf fest: "Das Pfarrblatt soll ein Forum sein: ein Diskussionspodium, bei dem auch einseitige Meinungen vorgetragen werden können. ${ }^{\alpha \theta}$

Um den Dialog in Gang zu bringen, empfiehlt sich oft die gezielte Veröffentlichung einseitiger, provozierender Artikel mit der Bitte an die Leser, dazu Stellung zu nehmen. Ein Beispiel aus jüngster Zeit findet sich im Basler Pfarrblatt, wo drei junge Paare, die unverheiratet zusammenleben, ausführlich Gelegenheit hatten, ihren Standpunkt darzulegen. Damit wirklich eine Diskussion zustande kam, wurde erst für 
eine spätere Nummer vorgesehen, den Standpunkt der kirchlichen Lehre zur Ehe darzulegen.

In einem Dialog-Forum ist es selbstverständlich, daß Leserbriefe, auch solche mit unkonventionellen Ansichten, veröffentlicht werden. Eine Umfrage unter den schweizerischen Pfarrblattredakteuren zeigte jedoch, daß die Leser im allgemeinen schreibfaul sind. So erhält beispielsweise der Redakteur des Zürcher Pfarrblatts mit einer Auflage von 50.000 durchschnittlich pro Nummer höchstens ein bis zwei Leserbriefe.

\section{Kontakte zu Fernstehenden}

Bekanntlich nimmt die Zahl der von der Kirche „Fernstehenden“ oder „Randsiedler“ immer mehr zu. Gleichzeitig ist das Angebot der durchschnittlichen Pfarre noch fast ausschließlich auf die Kerngemeinde ausgerichtet.

Aber auch wer an den kirchlichen Aktivitäten nicht mehr teilnimmt, kann für Fragen des Glaubens offen sein. Dies wurde in der Bundesrepublik durch die Synodenumfrage bestätigt, die zu dem Ergebnis kam, daß die Lektüre religiöser Publikationen nicht vom Kirchenbesuch oder vom Verhältnis zur Kirche abhängt ${ }^{7}$. Nur selten greifen jedoch „Randsiedler" ${ }^{“}$ zur kircheneigenen Presse. Denn diese haben noch allzu stark jene Christen als Zielgruppe, die ohnehin schon mit der kirchlichen Gemeinschaft in Kontakt stehen ${ }^{8}$. Ein redaktionelles Konzept, wie es beispielsweise der "Kirchenbote“ der reformierten Kirche des Kantons Zürich hat, ist selten: „Wir versuchen als ,Normalleser' gerade jene ins Auge zu fassen, die nicht auf ihre kirchliche Loyalität hin angesprochen werden können, sondern der institutionellen Kirche gegenüber zunächst einmal große Vorbehalte haben, ihr indifferent gegenüberstehen, mit ihr nichts anfangen können. ${ }^{* \theta}$

Hier liegt eine große Chance der Pfarrblätter. Sie können für viele das letzte und einzige Bindeglied zur Institution Kirche sein. Voraussetzung ist, daß der Leser nicht kirchliche Public relations vorgesetzt bekommt, sondern spürt: „Mea res agitur“.

Die Gratisabgabe des Pfarrblatts trägt dazu bei, daß es nicht bloß in die Hände jener gerät, die ohnehin schon am kirchlichen Leben interessiert sind. In der Schweiz besteht seit zwei bis drei Jahren die Tendenz, die Pfarrblätter allen katholischen Haushaltungen gratis zuzustellen. Dabei trägt man auch der Tatsache Rechnung, daß die Kirchensteuerzahler Anrecht auf eine Gegenleistung haben: „Die Steuerzahler haben das Recht, etwas von der Kirche zu bekommen, auch wenn sie ihren sakralen Service nicht mehr beanspruchen. Die Kirche als öffentliche Körperschaft hat zudem eine Informationspflicht. ${ }^{* 10}$

\section{Erwachsenenbildung und Lebenshilfe}

Viel Unsicherheit in der Kirche rührt daher, daß das religiöse Wissen bei manchen Gläubigen in keiner Weise Schritt gehalten hat mit der theologischen Entwicklung der letzten Jahre. Nicht wenige Christen sind auf der Stufe ihres Kinderglaubens stehen geblieben. Der Glaube ist für sie ein Relikt aus einer andern Entwicklungsstufe.

Gleichzeitig jedoch erwarten viele von der Kirche bzw. ihrer Presse Lebenshilfen, zum Beispiel in der Altenfrage, in Jugend- und Erziehungsfragen ${ }^{11}$. 
Wenn kirchliche Medien wie die Pfarrblätter auf solche Alltagsprobleme eingehen, dürfen sie selbstverständlich nicht bei Allerweltsweisheiten à la „Frau Brigitte“ stehenbleiben. Denn der Leser möchte wissen, wie er "seinen Lebenskampf sinnvoll, in Harmonie mit religiösen Werten bestehen kann"12.

Wenn die Pfarrblätter die Aufgabe von Erwachsenenbildung und Lebenshilfe übernehmen, genügt es nicht, dazu sporadisch und konzeptionslos Artikel zu veröffentlichen. Artikelfolgen und Lernziele sollten systematisch geplant werden. Nur ein gezieltes Vorgehen hat Erfolg. Deshalb müssen - wie in der Erwachsenenbildung allgemein - Schwerpunkte erarbeitet werden ${ }^{13}$.

\section{Sensibilisierung für gesellschaftliche Fragen}

Wie die Umfrage über die Kirchenpresse in der Bundesrepublik ergeben hat, werden an die Kirche hinsichtlich politischer und gesellschaftlicher Themen hohe Erwartungen gestellt: „Bis zu $81 \%$ der katholischen Gesamtbevölkerung erwarten regelmäßig oder gelegentlich Außerungen der Kirche zu Fragen der Altersversorgung, der Hilfe für sozial schwache Gruppen, bei Problemen der Schul- und Berufsbildung, in Erziehungsfragen, aber auch bezüglich Entscheidungen der Parlamente und Regierungen, in Steuer- und Gewerkschaftsfragen, in der Entwicklungshilfe für die Dritte Welt. ${ }^{14}$

Diesen Erwartungen entspricht das Selbstverständnis der Kirche. Wie schon im Alten Testament die prophetische Kritik gesellschaftliche Zustände am Gesetz Gottes gemessen hat, versteht sich auch die Kirche Jesu Christi als „ein auf die Gesamtgesellschaft bezogenes Medium" jenes Gottes, der seinen universalen Heilswillen kundgetan hat ${ }^{15}$. Ihre Aufgabe ist solidarische Mitsorge für Gegenwart und Zukunft der Menschheit. Wenn hier eine Domäne der Publizisten ist ${ }^{16}$, können auch die Pfarrblätter nicht an gesellschaftlichen Fragen vorbeigehen. Ihre Stärke liegt auch hier im lokalen Bereich. Durth nennt Beispiele, die in diesem Zusammenhang aufgegriffen werden können: Naherholung, Freizeit, Umweltschutz, Wohnqualität, Kultur, Bildung, ärztliche Versorgung, sozialer Interessenausgleich ${ }^{17}$.

In der Schweiz engagieren sich vor allem die größeren Pfarrblätter, zumal bei Volksabstimmungen, für menschlichere Lösungen in der Politik. Besonders war dies der Fall bei den Abstimmungen über die Gastarbeiter und über die Mitbestimmung.

\section{Folgerungen für das Pfarrblattwesen}

\section{Zusammenfassung der Aufgaben}

Bevor wir uns der Frage zuwenden, wie das Pfarrblattwesen organisiert sein muß, fassen wir die Hauptaufgaben der Pfarrblätter mit den Worten des Kommissionsberichts XII der St. Galler Synode zusammen:

"- Herstellung von menschlichen Verbindungen in überschaubarem regionalen und lokalen Raum;

- Information über Themen des religiös-theologischen Bereichs;

- Schärfung des Bewußtseins der Mitverantwortung;

- Förderung der aktiven Teilnahme am sozialen, kulturellen und politischen Leben durch die katholische Bevölkerung. ${ }^{\text {18 }}$ 
Konvergenzpunkt und Schwergewicht liegen hier wie bei unserer Darlegung offensichtlich bei der Integrationsfunktion des Mediums Pfarrblatt. Durch „Information“ soll „Kommunikation" geschaffen werden.

\section{Postulat: regionale Pfarrblätter}

Betrachtet man jede einzelne der skizzierten Aufgaben für sich wie auch in ihrer Gesamtheit, muß man feststellen: In der Regel kann nur ein Pfarrblatt, das von mehreren Pfarren einer Region gemeinsam herausgegeben werden, die genannten Funktionen einigermaßen befriedigend erfüllen. Wenn schon kantonale Blätter in der Schweiz Schwierigkeiten haben, genügend kompetente Mitarbeiter zu finden, stoßen Blätter einzelner Regionen sehr bald an die Grenzen personeller Möglichkeiten.

Trotzdem wehren sich in der Schweiz immer noch eine große Zahl von Pfarren gegen regionale Lösungen. Sie verteidigen "einen gesunden Individualismus“. An nicht wenigen Orten sperren sich auch die Druckereien, die bisher die Pfarrblätter einzelner Pfarren herstellten, gegen einen Zusammenschluß. Die Problematik solcher wirtschaftlicher Rücksichtnahmen zeigt das folgende Zitat eines Pfarrers: „Schon vor Jahren strebte ich ein kantonales Pfarrblatt an, das dann aber an den Druckereien scheiterte, die plötzlich behaupteten, der Druck des Pfarrblattes sei für sie eine Existenzfrage. Früher behaupteten sie immer, daß sie nichts verdienen ... ${ }^{\star 19}$

Bei aller Betonung der Aufgaben, die nur von einem regional hergestellten „Mantelteil “ befriedigend gelöst werden können, dürfen die pfarrlichen Belange nicht zu kurz kommen. Denn das Pfarrblatt ist und bleibt das Blatt der Pfarre! Deshalb ist „darauf zu achten, daß die Eigenart und Pfarrverbundenheit des einzelnen Pfarrblattes nicht einfach technischen Konzentrationsw ünschen geopfert wird“20.

Konkret bedeutet dies, daß in jedem Modell regionaler Zusammenarbeit jeder einzelnen Pfarre genügend Platz zusteht, den sie in eigener Verantwortung gestalten kann. Eine andere Frage ist dann, wie dieser Platz genutzt wird. In der Schweiz werden innerhalb der gleichen Region oft ganz verschiedene Erfahrungen gemacht. Es gibt Pfarrer, die sich mit den Gottesdienstzeiten begnügen und den Rest ihrer Pfarrseite(n) mit Füllern stopfen. Andere Pfarrer setzen sich nachdrücklich dafür ein, daß sie noch mehr Platz bekommen.

Die flexibelste Lösung für den Umfang der Pfarrseiten bietet das Luzerner Pfarrblatt an:

Variante A: Eine Pfarre verfügt über vier Pfarrseiten (ein Blatt eingeheftet), benutzt aber vielleicht nur drei Seiten (in diesem Fall: Seite 1 ein Klischee oder Pfarrtitel, oder Seite 4 wird leer gelassen);

Variante B: $Z$ wei oder drei Pfarren verfügen zusammen über vier Pfarrseiten;

Variante C: Drei oder mehr Pfarren verfügen zusammen über acht oder zwölf Pfarrseiten;

Variante D: Eine Pfarre verfügt in einzelnen Nummern über acht Pfarrseiten, d. h. bei besonderen Anlässen (z. B. Firmung) läßt der Pfarrer zwei Blätter einheften.

\section{Zusammenfassung}

Zum Abschluß versuchen wir zusammenfassend eine Definition der Pfarrblätter: In Abgrenzung zu „katholischen“ Zeitungen und Zeitschriften, die als „kirchennahe“ 
Medien bezeichnet werden, sind die Pfarrblätter „kircheneigene“ Medien" ${ }^{21}$. Im Unterschied zu den in vielen Zügen verwandten Bistumsblättern der BRD sind sie nicht auf das gesamte Bistum ausgerichtet, sondern (im Modell der Zusammenarbeit, das uns vorschwebt) auf regional umgrenzte Teile des Bistums beschränkt. Die Pfarrblätter sind somit kirchenbezogene, in der Regel kircheneigene, im lokalen und regionalen Raum verankerte Medien, die Integrationsfunktionen wahrnehmen, Forumscharakter tragen und sich in besonderem Maße auch an die Fernstehenden richten.

Anmerkungen:

1. Vgl. Clemens Kreuzer: Das Pfarrblatt als gemeindebildende Kraft. In: CS 6:1973, 193211.

2. A.a.O., 195.

3. Vgl. K. Rüdiger Durth: Lokalkommunikation der Kirchen. Der Pfarrer und die Zeitung. Remagen-Rolandseck 1975, 46.

4. Vgl. CeP, 115-117.

5. Vgl. Fritz Patrick Schaller: Zum Informationsrecht im kirchlichen Raum. Freiburg/ Schweiz 1970, $112 \mathrm{f}$.

6. Kurt Mahnig / Franz Wäger: Diskussionsgrundlage für ein Konzept des neuen Pfarrblattes. Bern 1972 (Manuskript), 2.

7. Vgl. Gerhard Schmidtchen: Zwischen Kirche und Gesellschaft, Forschungsbericht über die Umfragen zur Gemeinsamen Synode der Bistümer in der Bundesrepublik Deutschland. Freiburg 1972, 19 f.

8. Gottschlich kommt nach einer Analyse österreichischer Pfarrblätter zum Ergebnis: "Publizistisches Engagement zur Aktivierung speziell der Fernstehenden ist praktisch nicht erkennbar gewesen." Maximilian Gottschlich: Katholische Publizistik im sozialen Prozeß der lokalen Gesellschaft. Funktion und Erscheinungsbild österreichischer Pfarrblätter. (Diss., Manuskript) Wien 1973, 77.

9. Hans-Heinrich Brunner: Das Kirchenblatt im evangelischen Raum. In: „Schweizerische Kirchenzeitung“, 138:1970, 594.

10. Dekan Johann Stalder, Bern, im Gespräch mit dem Verfasser.

11. Vgl. Ferdinand Oertel: Informationsverhalten und -erwartungen der Katholiken. In: "Herder Korrespondenz“, 30:1976, 71.

12. Osterreichisches Pastoralinstitut (Hrsg.): Offentlidhkeitsarbeit der Pfarrgemeinde. System, Methoden, Praxis. Wien 1973, 108.

13. Für die Vielfalt der Möglichkeiten, ein Thema abzuwandeln, hier als Beispiel „Der gefangene Mensch": "1. Der Gefangene seiner selbst: Angst und Versagen; 2. Der Gefangene im sozialen Kontext: Familie, Schule, Beruf; 3. Der Gefangene von Systemen: Konsum, Werbung, Produktion, Massenmedien; 4. Der Gefangene seines Gewissens: Moral, Denkschablonen, Vorurteile, Verhaltensnormen; 5. Der Gefangene im Gefängnis: Recht, Strafe, Strafvollzug." Nach: Schweizerisches Pastoralsoziologisches Institut: Zielorientierte Seelsorge. Ziele und Wege für die pastorale Arbeit in Pfarrei und Region. St. Gallen 1975, 95.

14. Ferdinand Oertel: a.a.O., 70.

15. Vgl. Fritz Patrick Schaller: Notstand im christlichen Pressewesen. Sinn und Möglichkeit christlicher Pressearbeit, dargestellt an der Problematik der katholischen Presse in der Schweiz. (Diss.) Zürich, Einsiedeln, Köln 1974, 92-98 u. a.

16. „Die Propagierung der Werte der Freiheit, Gerechtigkeit, des Friedens, der Solidarität und der Liebe unter allen Menschen macht der Papst in eindringlichen Appellen den Publizisten zu einer genuin ihnen zufallenden Verpflichtung. " Giselbert Deussen in: Ethik der Massenkommunikation bei Papst Paul VI. Paderborn 1973, 153.

17. Vgl. Durth: a.a.O., 65 . 
18. Synode 72, Bistum St. Gallen: Information und Meinungsbildung in Kirche und Offentlichkeit. 6.1.1.

19. Zit. nach: Franz Thomann: Ergebnis der Umfrage über elf römisch-katholische Pfarrblätter der deutschsprachigen Schweiz. (Manuskript) Bern 1972, 10.

20. Synode 72, Bistum Basel: Information und Meinungsbildung in Kirche und Gesellschaft. 5.3.2.

21. Es gibt allerdings auch noch Pfarrblätter, die privaten Verlagen gehören. Sie sind jedoch zahlen- und bedeutungsmäßig im Abnehmen begriffen. Um jedoch auch ihnen Rechnung zu tragen, sprechen wir in der folgenden Definition von „kirchenbezogenen "Medien.

\section{S U M M A R Y}

The situation of Parish Bulletins in German-speaking Switzerland has changed since the'70s. Besides the very small leaflets, a considerable number of regional Parish Bulletins now appear in magazine style. These fulfil the role ascribed by pastoral theology and the sociology of communications to the Parish Bulletin. Being in a certain sense local newspapers, they contribute towards local integration. As a platform for discussion they help to prevent pluralism in the Church becoming polarised. In places where these bulletins are distributed free of cost they also help to unite those no longer taking an active part in parish life. Parish bulletins are also in the service of adult education, and can offer solutions to the problems in human life. Finally, they conscientize readers about their responsibilities to society.

\section{RÉ S U MÉ}

Depuis les années 70, le système des bulletins paroissiaux est en bouleversement en Suisse allemande. A côté des nombreuses petites feuilles sont apparus d'importants bulletins paroissiaux en »forme de magazines «. Ils remplissent - tout au moins au début - les fonctions qui peuvent être données par la pastorale et par la sociologie de communication aux bulletins paroissiaux: en tant que "journaux régionaux " dans le domaine ecclésiastique (paroissial), ils contribuent à l'intégration locale. En tant que forum de discussion, ils offrent leur concours pour que le pluralisme à l'intrérieur de l'Eglise ne mène pas à la polarisation. Surtout dans les lieux où ils viennent gratuitement dans tous les ménages catholiques, ils sont un trait d'union vers ceux qui $\mathrm{y}$ sont étrangers. Ils sont au service de la formation des adultes (formation de foi) et offrent une aide vitale. Finalement, ils sensibilisent les lecteurs à leur responsabilité dans le domaine social.

\section{R E S U M E N}

La hoja parroquial en la Suiza de habla alemana está en devenir desde el comienzo de los años 70 . Al margen de muchas hojas locales de pequeño formato, algunas de ámbito regional aparecieron en "formato de revista «. Cumplen - al menos aparentemente - con las funciones asignadas a las hojas parroquiales por la teología pastoral y la sociología de la comunicación social: contribuyen a la integración local como »periódicos locales« en el ámbito eclesial (parroquial). Como foro para la discursión contribuyen a evitar que el pluralismo eclesial interno degenere en polarización. Sobre todo en lugares donde llega gratis a todos los hogares católicos sirve de vínculo con los alejados. Están al servicio de la formación de adultos (adoctrinación en la fé)) y ofrecen ayuda vital. Por último, sensibilizan al lector para la responsabilidad en lo social. 